\title{
CONSIDERACIONES SOBRE 5 CASOS DE PURPURA FULMINANTE
}

\author{
Sindrome de Waterhouse-Friderichsen (*)
}

Por el Dr. ERICH SIMPFENDORFER

Ha querido la suerte que en el período del 11 de mayo al 14 de julio de 1942 tuviéramos la oportunidad de observar en ia Posta Infantil de Primeros Auxilios del Hospital M. Arriarán, una serie clínica de 5 casos de Púrpura Fulminante $y$ que bien pueden encuadrarse en el llamado Sindrome de Waterhouse-Friderichsen. El número de casos es considerable. ya que los casos, hasta abora publicados en la literatura universal no pasan más allá de 98 , según pudimos leerlo en un trabajo de Michael y Jacobus, publicado en el Archives of Pediatrics de marzo del presente año.

Al que expone le cupo el triste privilegio de atender personalmente a 4 casos de esta serie, y si digo triste, es por la fatalidad de un $100 \%$ de esta gravísima enfermedad y por tratarse de niños con un magnítico desarrollo pondo-estatural.

El cuadro que nos preocupa fué descrito por primera vez. por Völcker, en 1894; posteriormente por Little, en 1901: por Waterhouse, en 1911. y por Friderichsen, en 1918. Estos autores describieron una bemorragia bilateral de las glándulas suprarrenales asociada a un púrpura agudo fulminante. Este sindrome ha recibido el nombre de los dos últimos autores.

Aproximadamente el $70 \%$ de los 98 casos conocidos ocurrió en niños por debajo de los dos años y el $95 \%$ por debajo de los 9 años. En nuestra casuística, 2 casos están por debajo de los 2 años y 3 entre los 2 y 6 años.

(*) Entregado para se publicación el 3 de mayo de 1944. 


\section{Casuística.}

PRIMER CASO, - Obs, 46295.

J. B. M., 2 años, masculino, ingreas el 11 de mayo de 1942 a las 23 horas.

Ancecedentes herecitarios $y$ mórbidos: Sin importancia.

Enfermedad actu2: - Se inicia hoy en la mañana a las 11 boras con calofrios y decaimiento. Ha ptesentado deposiciones frecuentes, que ahora último se han tornado mucosas. Desde las 17 hotas presenta vomitos focuentes. En el tratascarso de la tarde le aparecen pequeñas petequias en todo el cuerpo, que van aumentando de tamaño.

Examen físico: Niño irritable y quejumbroso. Temperatura, 38.1. Pulso muy pequeño. Enfriamiento de las extremidades. Respiración quejumbrosa y entrecortada. Manchas porpúricas diseminadas por todo el cuerpo. de color violáceo. Inyección conjuntival intensạ bilatetal. Fsiquis embotado. Mucosa bacal igualmente con manchas purpúricas. Edema del labio superior. Pulmones despejados. Corazón con tonos apagados. Abdomen completamente depresible. No se palpa el bazo. Higado en el reborde costal.

Diagnóstico: Púrpura fulminante.

Tratamiento: Extracto suprarrenal 1 c.c.: vitamina K, 3 migrs.: calentamiento.

Evolución: Una hora dispaés del ingreso se aprecia un aumento considerable de las mancbas purpúricas en número $y$ tamañio $y$ el siño fallece at cotto rato.

Protocolo de autopsia (Drair. Peña): Maschas hemorrágicas de la piel de la frente, cuello, tronco, extremidades superiores y muslos, desde $3 \mathrm{~mm}$. de diámetro a $1 \mathrm{~cm}$. Cianosis de los labios y de las uñas. Hipethemia de la pía madre 7 del cercbro (se aprecia aplapamiento visible de las circunsvoluciones $\alpha$ rebiales. Degeneración granular del miocardio, bazo e hígado. Hipertrofia del timo y de los elementos linfáticos de la macosa intestinal. Hiperbemia intensa pulmonar bilateral. Hiperbemia de la mucosa faringea, laringea y esoffígica. Hemorragias an los ganglios mesentéricos. Degeneración granular y bemotragias pontiformes muy escasas de los riñones. Nî̃o entrófico.

Diagnéstico anátomo-patológico: Toxemia agadz. Hemorragia completa de ambas cápsulas supratrenates.

SROUNDO CASO. - Obs. 46537.

J. C. D., 4 años, masculino. Ingresa el 22 de mayo de 1942 a las 7 hores.

Antecedentes bereditarios: Sin importancia.

Enfermedad anterior: Sarampión benigno hace dos: meses.

Enfermedad actual: Se inicia ayer en la mañana con dolor localizendo a Ia pierna izquierda, por lo cual gnarda cama. Le dan fenalgina, A las 9 de 12 mañana decac bruscamente. Presenta gran astenia, vómitos y suspende au vida 
de melación. A media nocbe le notan sus familiatej la aparición de mancbas purpúricas en la cara $\mathbf{y}$ extremidades.

Expman físico: Semamente grave. Inconsciente. Pupilas iguales con escasa reacción laminos. Enfriamiento de las extremidades, Asfigmia. Cianosis de piel y mucasas. Exantema purpúrico de diferente tamaño, especialmente denso en la cara y extremidades inferintes. Tonos cardiacos apagados. No hay signos meringeos.

Diagnóstico: Púpura falminante. ¿Himorragia de las cápsulas suptarrenales?

Tratamiento: 'Extracto suptratrenal. 2 c.c.; suero clorurado al $10 \%, 20$ c.s.

Evolnción: Fallece 2 horas y 15 minutos después de su ingreso.

Protocolo de autopsia (Dra. Pé̃a): Lesiones parpníricas generalizadas de diferentes tamaños, aisladas en su mayoria confluentes. Hiperemia intensa de $l_{a}$ piamadre discreta del cerebro. Degeneración granular del miocardio. Hiperemia intensa pulmonat bilateral con no zona de eqplenización paravertebral inferior izquierda. Hiperplasia del timo que pesa 30 gts. Hiperplasia de los elementos linfáticos de toda la mucosa intestinal. Hiperplasio, hiperemia, y zonitas hemorrágicas escasas del bazo (pesa $110 \mathrm{grs}$ ). Degeneración granular e hiperemiar del higado y de los riñones. Hiperemia de la glándula suprarknal izquierda $y$ parcial de la detecha. No hay ksiones de los huesos.

Diagnóstico anàtomo-patológico: Toxemia aguda. Degeneración granular e hiperemia da los parénquimas.

TERCER CASO. - Obs. 48383.

CUARTO CASO. - M. V. R.. 1 año 8 meses, femenina. Ingrexa el 11 de julio de 1942 a las 12.30 horas.

A.ntecedentes bereditatios: Padres sanos, R. W. negativa; 7 hijos vivos. nno falkzido hace 6 dias de púrpura fulminante, a los 5 años de edad. Este cradro se inició a las 9 A. M. y el dzceso se produjo a las 10.30 A. M. deI mismo día. Como llegara en sstado agótsico a la Patta Infantil, no se hospitalizó.

Antecedentes personales: Sin importancia.

Enfermedades anteriores: Bronconeumonía a los 7 mesas; sarampión a las 12 meses.

Enfermedad actual: Se inicia ayer tarde con fiebre y desasosiego. No durmió en loda la noche. Hö́ en la mañina, a las 9 horas, le nota la madre la aparición de manchas purpúricas en la cara y el cuello, que van extendiéndose a las extremidades, acmentanòo zápidamente de tamaño. Anocbe ptesentó vómitos en 3 ocasiones.

Examen físico: Niño semi-inconsciente, con respiración quejumbrosa. Mirada vaga. Temperatura, 39.2\%. No se logra palpar el pulso radial" Man. chas purpútices exténsas y confluentes en la cara, cuello y extremidades st- 
periores. En el resto del cuerpo y en las extremidades inferiores se observari petequias. Cianosis de los labios y de la mucosa bucal. Faringe roja. Palmones despejados. Hígado a 2 traveses de dedo del réborde costal. Bazo no se palpa. No hay signos meningeos.

A pesar de no baber sigros meningeos se practida ponción lumbiar, que da sslida a L. C. R. completamente limpio.

Diagnóstico: Párpura fulminante. Menincococcemsia.

Tratamiento: Extracto suprartena!, l c.c. cada 4 brotas. Transfusión sanguinea, 80 c.e. Soludagenán. 1 amp. (l gr.) intravenosa en sueto fis. Sulfadiazina. 1 tableta cada 3 horas.

Exámenes de Labotatorio: Secreción nasal: Meningococo negativo, Secreción faríngea: Hay desarrollo de meningocosos. L. C. R.: Transparente con retícalo fíbrinoso. Albúmina: 0.25 . Clorutos: 7.41 . Glucosa: 0.45 , Glóbulos blancos: 8. Linfocitos: $100 \%$. Pandy: positivo.

Evolución: Fallece. a pesat del tratamiento, 4 horas y 15 mitutos después de su ingreso.

Protocolo de autopsia (Dra. Peña): Niña eutrófica. Zonzls bemortágicas numerosisimas desde $3 \mathrm{~mm}$. de diámetro hasta $1 \mathrm{~cm}$, de bondas irregulares en Ja cara, regiones glúteas y 4 extremidades (en ambos brazos son confluentes; irregulares, extensas, adoptando la forma de alas de mariposa): en las manos F los pies hay lesiones pápulo-hemorrágicas. Hiperemia intensa de la piamadre, menos internsa del cerebro (no se eneuentran lesiones de leptomeningitis). Degeneración granulat del miocardio. Hiperemia pulmonar bilaterai con zónas hemotrágicas hasta de $1 \mathrm{~cm}$. de diámetro en el lóbulo inferior dedecho. Hiperpiasia discreta de los elementos línfáticos de la última porción del ileon. Hiperemia y zonas hemorrigicas del bazo. Hemortagia de la totalidad de ambas glánúulas suprarrenàles, Degeneración granular e hiperemia intenss del hígado y de los riñones.

Diagnóstico anátomo-patológico: Toxemia. Hexorragia de las glándulas suptartenales.

QUINTO CASO. - Obs. 48699.

R. B. Y., 6 ẵos. fzmenina. Ingresa el 12 de julio de 1942 a las 7.30 boras.

Antecedentes tereditarios y mórbidos: Sin importancia.

Enfermedad actual: Hace 30 horas presenta un calofrío de un cuarto de hora de duración: hace 18 boras le notạn uparición de manchas quupurricas que van progtesivamente aumentando de tamario. en la cata, extremidades snperiores y después inferiores hasta hacerse confluentes en diversas partes: hace 10 horas presenta estado gauseoso y desde hace 8 horas la enferma entra en estado de inconsciencia, que persiste hasta su ingreso.

Examen físico: Enferma completamente incòscientr, quejumbrosa y ex* citada. Fuiso radial no se palpa. Temp., 38.5\%. Enfriamiento de las ex- 
teemidades. Manchas purpúticas extensas y confloentes en Ia cara y extremidades superiores e inferiores. El tronco esta relativamente respetado. Rigidez de la nuca. Signo de Brudzinski positivo, el de Kernig segativo. Trismus marcado que impide ver la faringe. Papilas desiguales con exasa reacción inminósa. Buzo no se palpa.

Punción Inmbar: L. C. R. opatesonte, prèsión algo aumertada.

Diagnóstico: Púrpura fulminante. Meningitis purulenta.

Tratamiento: Sulfadiazina, 6 tabletas iniciaies, seguir con 2 tabletas cada 3 horas.

Transfusión sanguinea de úrgencia, 100 c.s. Extracto supta-renal, 1 c.e. cada 3 hotas. Calentamiento.

Evolución: Después de la punción lambar, la enferma se tranquiliz.. A las pocas horas de inresada está sin polso, con respiración estertorosa y con psiquis totalmente abolido. Las manchas purpúricas han anmentado de tamañb. Fallece 9 horas después de su ingresa.

Eximenes de Laborstorio: L. C. R. Aspecto débilmente turbio, con grun retículo fibrinoso, Albúmina, 0.20. Glucosa, 0.04. Fandy ++++ . Cultivo: meningococo positivo.

Protocolo de autopsia (Dra, Peña): Léptomraningitis purulenta meningocócica del cerebro. Marcado aplenamiento de lsa circunvoluciones cerebrales; opacidad de la menioge $y$ exudado purulento escaso de las vainas perivaseulares de algunos vasos de la cara externa de ambos bemisferios; no bay hiperemia de la píamadre ni del cerebro. Degenetación granular del sriocardio y riñones. Hiperemia intensa pulmoinar bilateral. Hiperplasia discreta de los elementos linfáticos de la tnacosa de la fultima porción del ileon. Ascaridiosis. Hiperemia $y$ degeneración granular del bazo y del hígado. Hiperemia de las g!andulas supra-tenales, más intensa aún a la derecha, Manchas hemorrágicas ajsladas de medio a $1 \mathrm{~cm}$. de diámetto, de bordes itregulares, numerosas. en la cara, región dorsolumbar y las 4 extremidades. En las extremidades supet tiores, a nivel de los codos y en la cara interna de los mustos, son confluentes, alcanzando hasta $4 \mathrm{cms}$. de diámetro.

Expuestos los casos, cabe hacer algunas consideraciones:

En cuanto a etiología, según el trabajo de Michael y $J_{a}$ cobus, el $65-70$ \% de los casos son causados por el meningococo; el porcentaje restante se debe al bacilo de la influen$\mathrm{za}$, el neumococo, al estreptococo hemolítico y al estafilococo áureo.

En nuestros casos no cabe duda que el agente patógeno ha sido en todos ellos el meningococo; los 2 primeros casos, si bien es cierto que no se hicieron mayores investigaciones por su brevísima estada, se hacen muy sospechosos en un período de epidemia meningocócica. En el cuarto caso, en 
que se encontró el meningococo en la secreción faríngea y en que el L. C. R. ya mostraba una franca irritación meníngea, creo que las dudas son ínfimas con respecto a su etiología. El quinto caso salva toda posible duda, pues constituye el puente de unión entre el púrpura fulminante y la meningitis meningocócica, ya que al lado de un púrpura fulminante alcanza a desarrollarse una meningitis con cultivo- de meningococos positivo. Vale la pena tener presente que el último caso es el de mayor edad y el que sobrevivió mayor número de horas.

De los 4 casos relatados, 2 encuadran perfectamente en el sindrome de Waterhouse-Friderichsen; en los 2 restantes, si bien existía el cuadro clínico de un púrpura fulminante, faltaba la hemorragia de ambas cápsulas supratrenales. ¡Pero quién nos asegura que se hubiera presentado ésta en corto tiempo más, existiendo yà de hecho una intensa congestión de ellas?

Llama la atención la dramaticidad de todos los casos, su evolución tan breve y tan grave, en una palabra: fulminante. Se ha culpado a la constitución timo-linfática de ser la que ofrece el terreno para que se produzcan estos cuadros, y me permito recordar que en los 2 primercs casos había una hipertrofia del timo $y$ de los elementos linfáticos de la mucosa intestinal; en los 2 últimos, una hiperplasia discreta de los elementos linfáticcs de la misma mucosa. Sin duda, este estado constitucional debe jugar un papel importante en la hemorragia masiva de las glándulas supra-renales, más aun cuando se ha establecido que la hemorragia idiopática de éstas es mucho más frecuente observar en individuos de constituciỏn linfática.

Los autores americanos hacen mención a la falta de signos elínicos y anatómicos del sistema nervioso central y ello se debe a que los enfermos fallecen en el estado septicímico, antes que el cerebro y las meninges sean invadidas por el germen. En cuante a nuestros casos había, en el primer caso, un aplanamiento de las circunvoluciones cerebrales, indicador de una compresión intracraneana $y$, en el último, una leptomeningitis en comienzo; aun en el cuarto caso, el aumento de los elementos en el $\mathrm{L}$. C. $\mathrm{R}$. y la reacción de Pandy positiva, son indicadores de una inflamación que se inicia.

Diagnóstico: Cuadro purpúrica, brusco en aparición. con elementos purpúricos que aumentan rápidamente de ta- 
maño y número, con gran compromiso del estado general. unido al sindrome de insuficiencia aguda de las glándulas suprarrenales, deben hacer pensar siempre en el sindrome de Waterhouse-Friderichsen.

En cuanto a exámenes de laboratorio se ha encontrado en el hemograma una trombocitopenia. El hemecultivo, que debería practicarse en todos los casos, es de un gran valor, puesto que nos indica el germen causante. Si no se alcanza a practicar, los autcres aconsejan la punción cardíaca dentro de la primera media hora que sigue al deceso.

Tratamiento: Aunque todos los casos han sido fatales hasta ahora, no debe perderse tiempo en administrar extracto suprarrenal y sulfapiridina o sulfadiazina en dosis masivas, más aún cuando existen la sospecha de infección meningocócica. En los 2 últimos casos usamos la sulfadiazina a la dosis de $40 \mathrm{ctgrs}$. pnt kgr. de peso, al lado de transfusiones sanguineas.

Hay autores que recomiendan el uso de sangre por vía intramusculat, de veneno de cobra $y$ de vitamina $K$. 